\title{
ON RELATIONS AMONG RADICAL PROPERTIES
}

\author{
by A. D. SANDS
}

(Received 22 May, 1975; revised 5 September, 1975)

1. Introduction. In this paper we work in the class of associative rings. The fundamental definitions and properties of radicals may be found in Divinsky [1]. If $\alpha$ is a radical class of rings we shall denote the class of semi-simple rings by $\mathscr{S}_{\alpha}$; the radical of a ring $R$ we shall denote by $\alpha(R)$.

The following properties which a radical $\alpha$ may possess are considered:

$\alpha$ is said to be hereditary (left, right hereditary) if $\alpha$ is closed under ideals (left, right ideals) i.e. if $R \in \alpha$ and $A$ is an ideal (left, right ideal) of $R$ then $A \in \alpha$;

$\alpha$ is said to be left (right) strong if $R \in \mathscr{S}_{\alpha}$ implies that $R$ contains no non-zeto left (right) ideal belonging to $\alpha$.

Equivalent to the hereditary conditions is that every (left, right) ideal contained in the radical of a ring is radical. Equivalent to the strong conditions is that every radical left (right) ideal is contained in the radical of a ring.

There are other hereditary or strong conditions, such as properties for subrings, which can be considered but the above ones seem to be the most natural and we restrict our attention to them. Given the classical origin of the radical there is one other natural condition to consider, namely supernilpotence. We shall call a radical $\alpha$ supernilpotent if every nilpotent ring is radical. (Divinsky [1] also imposes the hereditary condition, but we shall consider these two conditions separately here.)

The purpose of this paper is to consider dependence and independence among the three hereditary and two strong conditions. Since it is clear that hereditary is implied by either left or right hereditary there remain twenty possible combinations of these conditions to consider. There is also a left-right duality, since, if $\alpha$ is a given radical, we may define its dual radical $\alpha^{\prime}$ by $R \in \alpha^{\prime}$ if and only if the opposite ring $R^{o p} \in \alpha$. So each result has a dual result, obtained by interchanging left and right. Thus there are thirteen sets of conditions, six of which are self-dual, to consider. Of these we show that four combinations of conditions are not possible; we give examples showing that seven of the other cases do arise, but we leave two cases undecided.

We use the following notations. If $R$ is a ring, $(1, R)$ denotes the usual over-ring with identity of $R ; R^{+}$denotes the ring with the same underlying additive abelian group as $R$, but with trivial multiplication. For suitable rings $A, B, C, D$ we use $\left[\begin{array}{ll}A & B \\ C & D\end{array}\right]$ to denote the set of all $2 \times 2$ matrices $\left[\begin{array}{ll}a & b \\ c & d\end{array}\right]$ with $a \in A, b \in B, c \in C, d \in D$; we consider only cases where matrix addition and multiplication are naturally defined in such a way that $\left[\begin{array}{ll}A & B \\ C & D\end{array}\right]$ forms a ring. $M_{n}(R)$ denotes the ring of $n \times n$ matrices with entries from a ring $R$. 
2. Dependence relations. The following result is essentially due to Krempa [4, Theorem 5] but he states it using both left and right strong.

LEMMA 1. If a radical is hereditary and left strong and $R$ is a radical ring so also is $R^{+}$.

Proof. Let $K=\left[\begin{array}{cc}(1, R) & (1, R) \\ 0 & 0\end{array}\right]$. Then $\left[\begin{array}{ll}R & 0 \\ 0 & 0\end{array}\right]$ is a radical left ideal of $K$ and so by the left strong condition is contained in the radical of $K$. Therefore $\left[\begin{array}{ll}R & 0 \\ 0 & 0\end{array}\right] K=\left[\begin{array}{ll}R & R \\ 0 & 0\end{array}\right]$ is contained in the radical of $K$. Since this is an ideal of $K$ it follows by the hereditary condition that $\left[\begin{array}{cc}R & R \\ 0 & 0\end{array}\right]$ is radical. $\left[\begin{array}{ll}0 & R \\ 0 & 0\end{array}\right]$ is an ideal of $\left[\begin{array}{cc}R & R \\ 0 & 0\end{array}\right]$. It follows that $R^{+} \cong\left[\begin{array}{cc}0 & R \\ 0 & 0\end{array}\right]$ is radical.

LEMMA 2. If the radical $\alpha$ is left hereditary and left strong it is right hereditary.

Proof. Let $R$ be a radical ring and let $A$ be a right ideal of $R$. Consider the ring $S$ of $2 \times 2$ matrices $\left[\begin{array}{cc}A & A \\ (1, R) & R\end{array}\right]$. By Lemma $1, R^{+}$is radical. $A^{+}$is an ideal of $R^{+}$and so is radical by the (left) hereditary condition. $\left[\begin{array}{ll}0 & A \\ 0 & R\end{array}\right]$ is an extension by $R$ of $A^{+}$and so is radical; it is a left ideal of $S$ and so, by the left strong condition, is contained in $\alpha(S)$. Hence $\left[\begin{array}{ll}0 & A \\ 0 & R\end{array}\right]\left[\begin{array}{cc}0 & 0 \\ (1, R) & 0\end{array}\right]=\left[\begin{array}{ll}A & 0 \\ R & 0\end{array}\right] \subset \alpha(S)$.

Since $\left[\begin{array}{ll}A & 0 \\ R & 0\end{array}\right]$ is a left ideal of $S$, it follows, using the left hereditary condition, that $\left[\begin{array}{ll}A & 0 \\ R & 0\end{array}\right]$ is radical. $A$ is a homomorphic image of this radical ring and so is radical. Therefore the radical $\alpha$ is right hereditary.

LEMMA 3. If the radical $\alpha$ is left hereditary and right strong then it is right hereditary.

Proof. Let $R$ be a radical ring and let $A$ be a right ideal of $R$. By the dual of Lemma 1, $R^{+}$is radical and so, as above, $A^{+}$is radical. Consider the ring $T$ of $2 \times 2$ matrices $\left[\begin{array}{ll}A & A \\ R & R\end{array}\right]$. $\left[\begin{array}{ll}0 & 0 \\ R & R\end{array}\right]$ is an extension by $R$ of $R^{+}$and so is a radical right ideal of $T$; hence, by the right strong condition, it is contained in $\alpha(T)$. Therefore

$$
\left[\begin{array}{ll}
0 & A \\
0 & R
\end{array}\right]\left[\begin{array}{ll}
0 & 0 \\
R & 0
\end{array}\right]=\left[\begin{array}{ll}
A R & 0 \\
R^{2} & 0
\end{array}\right] \subset \alpha(T)
$$

$\left[\begin{array}{ll}A R & 0 \\ R^{2} & 0\end{array}\right]$ is a left ideal of $T$ and so, by the left hereditary condition, it is radical. Hence $A R$ is a radical ideal of $A$. $A / A R$ is isomorphic to $A^{+} /(A R)^{+}$, since it has trivial multiplication. Thus it is an image of $A^{+}$and so is radical. Then $A$, being an extension of $A R$ by $A / A R$ is radical. It follows that $\alpha$ is right hereditary. 
LEMMA 4. If the radical $\alpha$ is left hereditary and left strong then it is right strong.

Proof. Let $R$ be a semi-simple ring. Let $A$ be a radical right ideal of $R$. Consider the ring $T$ of $2 \times 2$ matrices $\left[\begin{array}{ll}A & A \\ R & R\end{array}\right]$. By Lemma $1, A^{+}$is a radical ring. For each $r \in R,(r A)^{+}$ is a homomorphic image of $A^{+}$under the mapping $a \rightarrow r a, a \in A .(R A+A)^{+}$is a sum of these radical ideals $(r A)^{+}$together with $A^{+}$; thus $(R A+A)^{+}$is radical. Hence $\left[\begin{array}{cc}A & 0 \\ R A+A & 0\end{array}\right]$ is a radical left ideal of $T$; it follows, from the left strong condition, that it is contained in $\alpha(T)$. Therefore

$$
\left[\begin{array}{cc}
A & 0 \\
R A+A & 0
\end{array}\right]\left[\begin{array}{ll}
0 & A \\
0 & 0
\end{array}\right]=\left[\begin{array}{cc}
0 & A^{2} \\
0 & R A^{2}+A^{2}
\end{array}\right] \subset \alpha(T) .
$$

Since this is a left ideal of $T$ it follows, by the left hereditary condition, that it is radical. Thus its image, $R A^{2}+A^{2}$, is a radical ideal of $R . R$ is semi-simple and so contains no nonzero radical ideal. Therefore $A^{2}=0$. Then $A$ is a subideal of $R$, being an ideal of $A+R A$, which is an ideal of $R$. Therefore $A \subset \alpha(A+R A)=0$. It follows that the radical $\alpha$ is right strong.

THEOREM 1. If the radical $\alpha$ is either left or right hereditary and either left or right strong then it is left and right hereditary and left and right strong.

Proof. There are eight cases to consider. Three of these are covered in the previous three lemmas and three more by the dual results. The implication of right strong by right hereditary and left strong follows using the dual of Lemma 3 and then Lemma 4 . The remaining result is the dual of this one.

This theorem removes the possibility of four combinations of conditions (or their duals) holding. These are the combinations in which three of these conditions hold and the fourth does not and the combinations in which one hereditary and one strong condition hold but the others do not.

3. Constructions preserving radical properties. In this section we consider certain radical constructions which preserve some of these properties. These results are useful in providing examples illustrating that radicals do occur satisfying certain combinations of these conditions, but not the remaining conditions.

Since an intersection of radical classes is a radical class it is clear that the hereditary conditions are preserved under intersection. To show that this is also true for the strong conditions we need the following result whose proof was communicated to me, verbally, by W. G. Leavitt.

Lemma 5. If the radical $\alpha$ is left strong and the ring $R$ contains a radical left ideal $L$ then the ideal $I$ of $R$ generated by $L$ is radical.

Proof. Since $\alpha$ is left strong and $L$ is a radical left ideal of $I$ it follows that $L$ is contained 
in $\alpha(I)$. However $\alpha(I)$ is an ideal of $R$. From $L \subset \alpha(I) \subset I$ it follows that $\alpha(I)=I$. Therefore $I$ is radical.

THEOREM 2. If $\alpha_{i}$ is a family of left strong radicals and the radical $\alpha$ is defined by $\alpha=\cap \alpha_{i}$ then $\alpha$ is a left strong radical.

Proof. Let $R$ be an $\alpha$-semi-simple ring and let $L$ be an $\alpha$-radical left ideal of $R$. Then $L \in \alpha=\cap \alpha_{i}$ and so $L$ is $\alpha_{i}$-radical for each $i$. By Lemma 5 the ideal $I$ of $R$ generated by $L$ is $\alpha_{i}$-radical for each $i$. Therefore $I \in \cap \alpha_{i}=\alpha$, i.e. $I$ is $\alpha$-radical. Since $R$ is $\alpha$-semi-simple it follows that $I=0$ and so that $L=0$. Therefore $\alpha$ is a left strong radical.

The union of radical classes is not, in general, a radical class. However it follows from known results (see [3], [5], [6]) that if a class of rings satisfies one of the hereditary conditions so does the lower radical generated by it. Thus, if $\alpha_{i}$ is a family of radicals satisfying any of these hereditary conditions, the lower radical $\alpha$ generated by $\cup \alpha_{i}$ does so also.

The corresponding result does not hold for left strong radicals. It follows immediately from Theorem 2 that a least left strong radical containing a given class of rings exists; a construction for this radical has been given in [2]. We use this construction to show that if $\gamma$ is the lower radical generated by the union of radical classes $\alpha$ and $\beta$, where $\alpha$ and $\beta$ are left strong radicals, then $\gamma$ need not be left strong.

Let $\alpha$ be the lower left strong radical generated by a field $F$ of prime order. Let $\beta$ be the lower Baer radical; $\beta$ is known to be left strong. Let $\gamma$ be the lower radical generated by $\alpha \cup \beta$. Consider the ring $R=M_{2}(F) . \quad R$ contains a left ideal $L=\left[\begin{array}{ll}F & 0 \\ F & 0\end{array}\right]$ which is an extension by $F$ of $F^{+} . F \in \alpha \subset \gamma$ and $F^{+} \in \beta \subset \gamma$. Therefore $L$ is $\gamma$-radical. $R$ is $\beta$-semi-simple. We complete the proof that $\gamma$ is not left strong by showing that $R$ is also $\alpha$-semi-simple and so $\gamma$-semi-simple. Since $R$ is a simple ring if it is not $\alpha$-semi-simple, it is $\alpha$-radical. We use the notation of [2, p. 375] with $\mathscr{M}_{1}=\{F, 0\}$ and working with left ideals rather than right ideals. If $R$ is $\alpha$-radical then there exists a least ordinal $u$ with $R \in \mathscr{M}_{u}$. Clearly $u>1$. Therefore $R$ contains a proper non-zero left ideal $A$ in $\mathscr{M}_{v}$ for some $v<u$. The only proper nonzero left ideals are minimal, by the Wedderburn-Artin structure theorem, and are isomorphic to $\left[\begin{array}{ll}F & 0 \\ F & 0\end{array}\right]$. Again clearly the least such $v$ is greater than 1 . Thus $\left[\begin{array}{ll}F & 0 \\ F & 0\end{array}\right]$ contains a proper non-zero left ideal $B$ in $\mathscr{M}_{w}$ for some $w<v$. $\left[\begin{array}{ll}0 & 0 \\ F & 0\end{array}\right]$ is the only proper non-zero left ideal of $A . B$ is not in $\mathscr{M}_{1}$, but contains no proper non-zero left ideal. This contradicts $B \in \mathscr{M}_{w}$. Therefore $R$ is $\alpha$-semi-simple and $\gamma$ is not left strong.

This example does not cover the corresponding left and right strong result. $A$ contains $\left[\begin{array}{ll}F & 0 \\ 0 & 0\end{array}\right]$ as a right ideal and so in fact $R \in \mathscr{N}_{3}$, in the notation of [2]. This shows that the lower (left and right) strong radical construction can produce simple rings in the constructed radical class, not already in the original class, unlike the lower radical construction. It leaves open the question as to whether the lower radical generated by a union of strong radical classes is strong. 
4. Examples of radicals. In this section we give a list of radicals satisfying certain combinations of the hereditary and strong conditions. In each case we try to give examples showing that the supernilpotent condition is independent. Many of these examples are well known and we omit routine verifications.

EXAMPLE 1. Radicals satisfying all these hereditary and strong conditions. The Jacobson, Levitzki and lower Baer radicals are known to satisfy all five conditions and are supernilpotent. The torsion radical (a ring $R$ is radical if and only if each element of $R$ has finite order under addition) satisfies all five conditions and is not supernilpotent.

EXAMPLE 2. Radicals satisfying all the hereditary conditions but not the strong conditions. If $F$ is a field of prime order then the lower radical generated by $\left\{F, F^{+}\right\}$satisfies all the hereditary conditions. It is not left or right strong since $M_{2}(F)$ is semi-simple but contains radical left and right ideals $\left[\begin{array}{ll}F & 0 \\ F & 0\end{array}\right]$ and $\left[\begin{array}{ll}F & F \\ 0 & 0\end{array}\right]$ (Leavitt [5]). This radical is not supernilpotent. The lower r adical generated by $F$ and all nilpotent rings is supernilpotent and retains the hereditary conditions, while the same example shows that it is not left or right strong.

EXAMPLE 3. Radicals which satisfy the strong conditions but not the hereditary conditions. Leavitt [5] gives the idempotent radical (a ring $R$ is radical if and only if $R^{2}=R$ ) as an example of a left and right strong radical which is not hereditary. This radical is not supernilpotent; indeed the nilpotent rings are semi-simple. A supernilpotent radical with these properties may be obtained as follows: let $\alpha$ be the upper radical generated by the proper ideals of $Z$ i.e. the rings $n Z, n>1$. By Theorem 3 of [8] $\alpha$ is left and right strong. No non-zero image of $Z$ is a subideal of this class of rings $\{n Z, n>1\}$ and so $Z$ is $\alpha$-radical. Hence the radical $\alpha$ is not hereditary. Similarly every nilpotent ring is $\alpha$-radical and so $\alpha$ is supernilpotent.

EXAMPLE 4. Radicals which satisfy the strong conditions and the hereditary condition but not the left or right hereditary conditions. If $F$ is a field then the upper radical $\alpha$ generated by $F$ is a special radical and so is hereditary. By Theorem 3 of [8] this radical is left and right strong. The ring $M_{2}(F)$ is radical but its left and right ideals $\left[\begin{array}{cc}F & 0 \\ F & 0\end{array}\right]$ and $\left[\begin{array}{cc}F & F \\ 0 & 0\end{array}\right]$ have $F$ as an image and so are not radical. Hence the radical $\alpha$ is not left or right hereditary. $\alpha$ is a supernilpotent radical. The intersection of $\alpha$ with the torsion radical remains hereditary and, by Theorem 2, remains left and right strong. The example shows that it is not left or right hereditary, provided that the field $F$ is chosen with prime characteristic. This radical is not supernilpotent, since a torsion-free nilpotent ring is not radical.

EXAMPLE 5. Radicals which are hereditary but not left or right hereditary nor left or right strong. The Brown-McCoy radical is hereditary. It is not left or right strong [2] nor left or right hereditary [7]. The Brown-McCoy radical is supernilpotent. The example given in [2] to show that it is not strong uses essentially a field of characteristic zero and it is not clear if the intersection of the Brown-McCoy radical with the torsion radical affords a nonsupernilpotent radical of the required type. The following is an example of such a radical. Let $F$ be a field of prime order. Let $\alpha$ be the lower radical generated by $M_{2}(F)$ and $F^{+}$. 
Then $\alpha$ is hereditary. The left and right ideals $\left[\begin{array}{cc}F & 0 \\ F & 0\end{array}\right]$ and $\left[\begin{array}{ll}F & F \\ 0 & 0\end{array}\right]$ of the radical ring $M_{2}(F)$ are not radical since they have the semi-simple-ring $F$ as an image. Hence $\alpha$ is not left or right hereditary. The left ideal $L$ of the semi-simple ring $M_{3}(F)$ is radical, since it contains an ideal $A$ isomorphic to $F^{+} \oplus F^{+}$, which is radical, and $L / A$ is isomorphic to $M_{2}(F)$, which is radical, where

$$
L=\left[\begin{array}{lll}
F & F & 0 \\
F & F & 0 \\
F & F & 0
\end{array}\right] \text { and } A=\left[\begin{array}{lll}
0 & 0 & 0 \\
0 & 0 & 0 \\
F & F & 0
\end{array}\right]
$$

Hence $\alpha$ is not left strong and a similar example shows that it is not right strong. $\alpha$ is not supernilpotent since $G^{+}$is semi-simple, where $G$ is any field of characteristic different from that of $F$.

EXAMPLE 6. A radical which is left strong but not right strong and not hereditary. In [8] it is shown that the upper radical generated by $\left[\begin{array}{ll}F & 0 \\ F & 0\end{array}\right]$ and $F^{+}$, where $F$ is a field of prime order, is left strong but not right strong. To see that this radical is not hereditary consider the ring $S=\left[\begin{array}{cc}F & F \\ 0 & 0\end{array}\right] . S$ is radical since neither $S$ nor its only non-zero image $F$, contains a non-zero subideal from $\left\{\left[\begin{array}{ll}F & 0 \\ F & 0\end{array}\right], F^{+}\right\} \cdot\left[\begin{array}{ll}0 & F \\ 0 & 0\end{array}\right]$ is a semi-simple ideal of $S$. This radical is not supernilpotent, since $F^{+}$is semi-simple. We have not been able to find a supernilpotent radical with these properties.

EXAMPLE 7. A radical satisfying none of these hereditary or strong properties. It seems reasonable to expect that a randomly chosen class of rings will generate a lower radical satisfying none of these conditions and that a randomly chosen class containing all nilpotent rings will generate such a supernilpotent radical. One possible choice in the first case is $\left\{F,\left[\begin{array}{ll}F & 0 \\ F & 0\end{array}\right],\left[\begin{array}{cc}F & F \\ 0 & 0\end{array}\right]\right\}$ where $F$ is a field. The lower radical is not hereditary since $F^{+}$is semisimple but is isomorphic to an ideal in $\left[\begin{array}{ll}F & 0 \\ F & 0\end{array}\right]$ and it is not left or right strong since $M_{2}(F)$ is semi-simple but contains radical left and right ideals. However we have not found such a class in the supernilpotent case, or, at least, we have not been able to show that it is such a class. Instead we consider the upper radical generated by a well-known simple ring without identity. Let $R$ be the ring of row-finite matrices, indexed by the set of positive integers, with entries from a field $F$. Let $S$ be the set of matrices of $R$ such that each matrix has only a finite number of non-zero columns. Then $S$ is the only proper non-zero ideal of $R$ and $S$ is a simple ring without identity. Let $\alpha$ be the upper radical generated by $S$. Every nilpotent ring is radical; so $\alpha$ is supernilpotent. $R$ is radical; so $\alpha$ is not hereditary. To see that $\alpha$ is neither left nor right strong we exhibit radical left and right ideals in $S$. Let $L$ be the set of all matrices in $S$ all of whose columns are zero except perhaps the first. Then the set $A$ 
of matrices in $L$ with zero in position $(1,1)$ is a nilpotent ideal of $L$ and $L / A$ is isomorphic to $F$. Therefore $A$ and $L / A$ are radical. Thus $L$ is a radical left ideal of $S$. Similarly the set $M$ of all matrices of $S$ all of whose rows are zero except perhaps the first is a radical right ideal of $S$.

There remain two unsolved cases (with their duals). Firstly we have the problem of whether a left hereditary radical must be right hereditary and secondly the problem of whether a hereditary radical which is left strong must be right strong.

\section{REFERENCES}

1. N. J. Divinsky, Rings and radicals (Allen and Unwin, 1965).

2. N. J. Divinsky, J. Krempa and A. Sulinski, Strong radical properties of alternative and associative rings, J. Algebra 17 (1971), 369-388.

3. A. E. Hoffman and W. G. Leavitt, Properties inherited by the lower radical, Portugal Math. 27 (1968), 63-66.

4. J. Krempa, Radicals of semi-group rings, Fund Math. 85 (1974), 57-71.

5. W. G. Leavitt, Lower radical constructions, Colloq. Math. Soc. Janos Bolyai 6 (1973), 319-324.

6. R. F. Rossa, More properties inherited by the lower radical, Proc. Amer. Math. Soc. 33 (1972), 247-249.

7. A. D. Sands, Radicals and Morita contexts, J. Algebra 24 (1973), 335-345.

8. A. D. Sands, Strong upper radicals, Quart. J. Math. Oxford (2) 27 (1976), 21-24.

Department OF Mathematics

THE UNIVERSITY

DUNDEE

DD1 4HN

SCOTLAND 